\title{
Radiation spectra from MHD simulations of low angular momentum flows
}

\author{
Monika A. Moscibrodzka, ${ }^{1}$ Daniel Proga,${ }^{2}$ Bożena Czerny ${ }^{1}$ \\ and Aneta Siemiginowska ${ }^{3}$ \\ ${ }^{1}$ N. Copernicus Astronomical Center, Bartycka 18, 00-716, Warsaw, Poland ${ }^{2}$ University of \\ Nevada, Las Vegas, Las Vegas, United States \\ ${ }^{3}$ Harvard-Smithsonian Center for Astrophysics, 60 Garden Street, Cambridge, MA 02138, USA \\ email: mmosc@camk.edu.pl
}

\begin{abstract}
We perform Monte Carlo calculation to determine the radiation spectra from magnetized, low angular momentum accretion flow. Magneto-hydrodynamical simulation (with angular momentum parameter lambda of about $2 \mathrm{Rg} \mathrm{c}$ ) was performed by Proga \& Begelman 2003. Because simulation neglect radiative cooling, we compute electron temperature separately, including ohmic heating, parametrized with small $\delta$ coefficient, ion-electron coupling, radiative cooling and advection. Radiation spectra are obtained taking into account, thermal synchrotron and bremsstrahlung radiation, self absorption and Comptonization processes.

We show which parts of the flow are responsible for characteristic spectral features and how the spectrum changes in different accretion states. We compare our results with Galactic Center black hole radiation spectra, where low angular momentum accretion is suggested. Accretion state changes seems to be a promising model for the flaring behavior of Galactic Center black hole. We also show the radiation intensity maps in radio and X-rays energy band for viewing angle $\mathrm{i}=90^{\circ}$.
\end{abstract}

Keywords. Accretion, accretion disks - magnetic fields - (magnetohydrodynamics:) MHD Galaxy: center

\section{Modeling}

To perform Monte Carlo simulation, we follow the algorithms given by Pozdnyakov et al. 1983 and Gorecki \& Wilczewski 1984. We have chosen two characteristic accretion states in late time of evolution of the accretion flow. First one, when evolved torus (extending to the last stable orbit) accrete (T1), and the second one, when the accretion is stopped (T2) due to strong magnetic field that forms a magnetized polar cylinder around the black hole (Fig. 1, on the left). Moments of time in simulation: T1 and T2 are measured in units of Keplerian orbital time at $r=R_{B}$ (Bondi radius) (for Sgr A* $\mathrm{T} 2-\mathrm{T} 1=0.02 \times A[\mathrm{~s}], A=2 \pi R_{B} / \sqrt{G M / R_{B}} \sim 0.32$ [year], thus T2-T1 $\sim 2.3$ [days]).

We rescaled density and magnetic field in purpose to test the model in case of Sgr A*. Scaling factor was chosen to fit the mass accretion rate at the outer and inner boundary of the flow (limit for mass accretion rate at inner boundary is superimposed by polarization measurements). Our calculations show that synchrotron photons are created mainly in a magnetized corona of the torus very close to the black hole. Bremsstrahlung radiation comes out from outer regions (Fig. 1, on the right). Because only inner flow changes, the variability can be seen just in synchrotron and Comptonization energy range. We computed broad band radiation spectra (Fig. 2, on the left) and intensity maps for two cases $\mathrm{T} 1$ and $\mathrm{T} 2$ (in radio and X-ray bands), when the observer is situated near the equator $\left(i=90^{\circ}\right)$ (Fig. 2, on the right). 

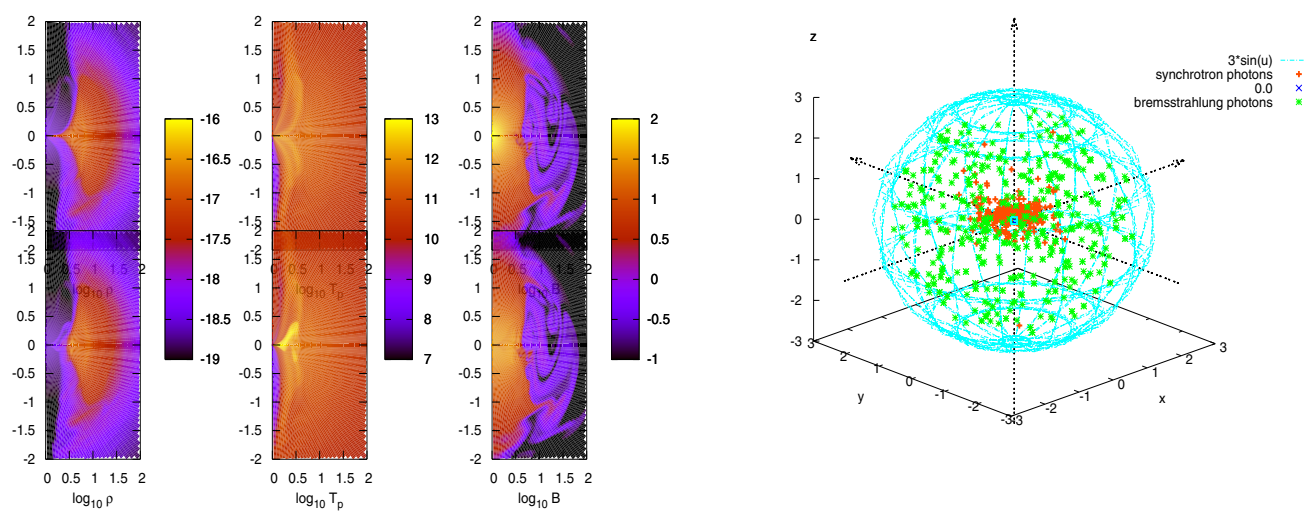

Figure 1. Density,temperature and mean magnetic field maps for two-accretion states T1 (upper panels) and T2 (lower panels) (on the left). 3-D map of synchrotron and bremsstrahlung photons birth place for one of the accretion states (radius is given in $\log R_{g}$ units) (on the right).
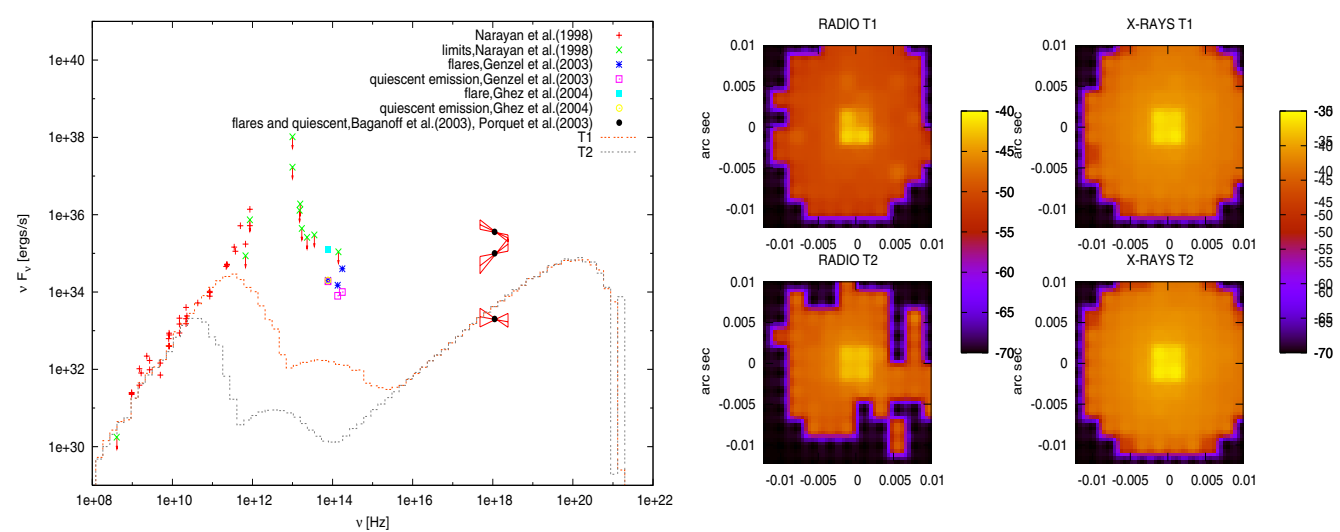

Figure 2. Broadband radiation spectra for two accretion states T1 and T2 calculated for Galactic Center black hole (on the left) Radio $\left(10^{12} \mathrm{~Hz}\right)$ and X-ray $\left(10^{17} \mathrm{~Hz}\right)$ pictures for two accretion states for observer at $i=90^{\circ}$ (on the right).

\section{The case of Sgr A*}

'Quasi-stationary' Sgr A* radiation spectrum cannot be fitted satisfactory by neither of accretion states due to a weak magnetic field. Changes in radio-IR level of radiation can indicate that similar process can be responsible for radio-IR flaring in Sgr A*, however emission level, predicted by the model, is much too weak at these energies. Low energy radio tail is fitted very well to the observed points. At higher energies (X-ray band) the spectrum does not vary because bremsstrahlung photons are born mostly in outer parts of the flow. X-ray flaring must be created by non-thermal process, or another accretion state (see Proga \& Begelman 2003).

\section{References}

Gorecki, A. \& Wilczewski, W. 1984, AcA, 34, 141

Pozdnyakov, L. A., Sobol, I. M. \& Syunyaev, R. A. 1983, ASPR, 2, 189

Proga, D. \& Begelman, M. C. 2003, ApJ, 592, 767 